Check for updates

Cite this: RSC Adv., 2018, 8, 29775

\title{
Tracking intramolecular energy redistribution dynamics in aryl halides: the effect of halide mass $\uparrow$
}

Received 11th June 2018

Accepted 16th August 2018

DOI: $10.1039 / \mathrm{c} 8 \mathrm{ra04979c}$

rsc.li/rsc-advances

\author{
Xiaosong Liu, (D) a Yunfei Song, t' $^{\mathrm{b}}$ Wei Zhang, ${ }^{\mathrm{a}}$ Gangbei Zhu, ${ }^{\mathrm{b}}$ Zhe Lv, Weilong Liu*a \\ and Yanqiang Yang*ab
}

Selective excitation of $\mathrm{C}-\mathrm{H}, \mathrm{C}-\mathrm{C}, \mathrm{CX}^{1}$ and $\mathrm{CX}^{2}$ stretching vibrational modes in an orderly manner, detection of intramolecular energy redistribution and vibrational coupling in the electronic ground state of aryl halides are performed by time- and frequency-resolved Coherent Anti-Stokes Raman Scattering (CARS) spectroscopy. Intramolecular energy flow from parent modes to daughter modes is observed in the experiment. According to the experimental results, it is found that the up-hill vibrational energy flow from lower frequency modes to higher frequency ones is counterintuitive and energy redistribution efficiencies are controlled by the mass of the halide. The selectivity and directionality of energy flow are also discussed in view of vibrational symmetry.

\section{Introduction}

The substituent effect is one of the most important concepts in chemistry, biochemistry, and related fields. ${ }^{1-4}$ When substituted benzenes became the subject of a spectroscopic study, ${ }^{5-8}$ there was much confusion regarding the results, such as the influence of substituents on reactivity and stability of the very wide range of organic species containing aromatic moieties. ${ }^{\mathbf{9 - 1 2}}$ Analysis of mono-substituted benzenes reveals that the influence of a single group will be essential. ${ }^{11,12}$

Monosubstituted benzenes are chosen for this study because of our prior knowledge of benzene's intramolecular energy redistribution, ${ }^{\mathbf{1 3}}$ and we can describe a series of molecules where the basic phenyl structure remains unchanged. According to our previous work, ${ }^{13}$ isolated-molecule energy flow of benzene was described, so the vibrational energy redistribution observed in liquid aryl halides $\mathrm{X}-\mathrm{Ph}(\mathrm{X}=\mathrm{F}, \mathrm{Cl}, \mathrm{Br}, \mathrm{I} ;-\mathrm{Ph}=$ $\mathrm{C}_{6} \mathrm{H}_{5}$ ) is entirely a consequence of intramolecular interactions. Using the narrow band CARS technique, it is easy to understand the effect of substituent mass on vibrational energy redistribution. We see two prominent ring stretching modes, $\nu_{\mathrm{CH}}$ $\left(\sim 3070 \mathrm{~cm}^{-1}\right), \nu_{\mathrm{CC}}\left(\sim 1580 \mathrm{~cm}^{-1}\right)$, whose frequencies in benzene and in aryl halides are quite similar, along with two substituentdependent modes of $\nu_{\mathrm{CX}^{1}}\left(\sim 1080 \mathrm{~cm}^{-1}\right)$, and $\nu_{\mathrm{CX}^{2}}\left(\sim 700 \mathrm{~cm}^{-1}\right)$. In subsequent comparisons of intramolecular energy

${ }^{a}$ Department of Physics, Harbin Institute of Technology, Harbin, China

${ }^{b}$ National Key Laboratory of Shock Wave and Detonation Physics, Institute of Fluid Physics, China Academy of Engineering Physics, Mianyang, China. E-mail: yqyang@ caep.cn

$\dagger$ Electronic supplementary information (ESI) available. See DOI: 10.1039/c8ra04979c

\$ This author contributed equally to this work and should be considered co-first authors. redistribution within the series of aryl halides $\mathrm{F}, \mathrm{Cl}, \mathrm{Br}$ and $\mathrm{I}$, we will refer to increasing mass, that means halide substitution can affect the vibrational dynamic via mass effects.

In this work, vibrational modes of phenyl and substituentdependent are selectively excited, and the normal intramolecular energy flow from high frequency phenyl modes to neighbouring bands and the counterintuitive up-hill energy flow from substituent-dependent modes to higher phenyl modes are detected. Quantum beats among these relevant modes in the vibrational ground state are observed, and the vibrational coupling information is extracted via Fourier analysis. The effects of halide mass on the efficiencies of intramolecular energy redistribution are described.

\section{Experimental}

The light source we used is a Ti:sapphire laser system (SpectraPhysics). The duration, repetitive frequency, single pulse energy, and center wavelength of the pulse generated from a regenerative amplifier (Spitfire, Spectra-Physics) are $110 \mathrm{fs}, 1$ $\mathrm{kHz}, 500 \mu \mathrm{J}$ and $800 \mathrm{~nm}$, respectively. The output beam is split by a $9: 1$ beam splitter, then the beam with 90\% energy is focused into an optical parametric amplifier (OPA) and the output pulse is further split into two beams that are used as pump and probe pulses; the other beam with $10 \%$ energy is served as the Stokes pulse (800 nm, FHWM: $13.4 \mathrm{~nm}, 795-808$ $\mathrm{nm}$ ). Photon energy difference between the Stokes and pump pulses is tuned by changing the center wavelength of the pump pulse, which can achieve the selective excitation of a particular vibrational mode. Specifically, the center wavelengths of the pump and probe pulses are changed from 763 to $645 \mathrm{~nm}$, the center wavelength of the Stokes pulse is fixed at $800 \mathrm{~nm}$. The Raman active vibrational modes $\nu_{\mathrm{CX}^{2}} \sim 700 \mathrm{~cm}^{-1}, \nu_{\mathrm{CX}^{1}} \sim$ 
$1080 \mathrm{~cm}^{-1}, \nu_{\mathrm{CC}}$ (in range from $1320 \mathrm{~cm}^{-1}$ to $1590 \mathrm{~cm}^{-1}$ ) and $\nu_{\mathrm{CH}}$ $\sim 3070 \mathrm{~cm}^{-1}$ are selectively excited. Regarding the beam geometry, the folded BOXCAR configuration with properly chosen angles between the beams defined by the phasematching condition is employed. Thus, the CARS signal is spatially separated from the other beams. The detection setup consists of a spectrometer (Bruker Optics 500 IS/SM) and an intensified charge coupled device (ICCD, Andor DU440-BU2).

\section{Results and discussion}

\section{Vibrational mode assignments}

The vibrational modes of aryl halides in our work are essentially in agreement with the experimental assignments of the vibrational spectra, ${ }^{\mathbf{1 0 1 4 - 1 7}}$ we group them into three categories, namely, the phenyl modes, the substituent modes, and the combination bands. The vibrational mods of theory calculation results by Gaussian 03 are listed in ESI (Tables 5-8†). The visible modes in the CARS experiments are sorted as follows:

(1) Phenyl modes: the $\nu_{\mathrm{CH}}\left(\sim 3070 \mathrm{~cm}^{-1}, A_{1}\right.$ or $B_{1}$ symmetry); $\nu_{\mathrm{CC}}$ (in range from $1320 \mathrm{~cm}^{-1}$ to $1590 \mathrm{~cm}^{-1}, A_{1}$ or $B_{1}$ symmetry); $\beta_{\mathrm{CH}}$ (in range from $1000 \mathrm{~cm}^{-1}$ to $1290 \mathrm{~cm}^{-1}, A_{1}$ or $B_{1}$ symmetry); $\gamma_{\mathrm{CH}}$ (in range from $730 \mathrm{~cm}^{-1}$ to $980 \mathrm{~cm}^{-1}, A_{2}$ or $B_{2}$ symmetry); $\phi_{\mathrm{CC}}\left(\sim 600 \mathrm{~cm}^{-1}, B_{2}, B_{1}\right.$ or $A_{2}$ symmetry).

(2) Substituent-dependent modes: $\nu_{\mathrm{CX}^{1}}\left(\sim 1080 \mathrm{~cm}^{-1}, A_{1}\right.$ symmetry), $\nu_{\mathrm{CX}^{2}}$ ( $\sim 700 \mathrm{~cm}^{-1}, A_{1}$ symmetry).

(3) Combination bands: $\nu_{\mathrm{CM}^{1}} \sim 2230 \mathrm{~cm}^{-1}\left(B_{1}\right.$ symmetry, $\nu_{\mathrm{CX}^{1}}$ $\left.\sim 1080 \mathrm{~cm}^{-1}+\beta_{\mathrm{CH}} \sim 1150 \mathrm{~cm}^{-1}\right) ; \nu_{\mathrm{CM}^{2}} \sim 2500 \mathrm{~cm}^{-1}\left(A_{1}\right.$ symmetry, $\left.\nu_{\mathrm{CC}} \sim 1450 \mathrm{~cm}^{-1}+\beta_{\mathrm{CH}} \sim 1066 \mathrm{~cm}^{-1}\right){ }^{10,14}$

\section{Selective excitation of the $\mathrm{C}-\mathrm{H}$ stretching vibrational modes}

Selective excitation of high frequency $\mathrm{C}-\mathrm{H}$ stretching vibrational modes and detection of vibrational energy redistributes to lower frequency combination bands are shown in Fig. 1. The white lines are the experimental spontaneous Raman spectra of aryl halides. Vibrational modes in the frequency range from $2752 \mathrm{~cm}^{-1}$ to $3298 \mathrm{~cm}^{-1}$ are selectively excited by a pair of pump (642 nm, FHWM: $14 \mathrm{~nm}, 638-652 \mathrm{~nm}$ ) and Stokes pulses (800 nm, FHWM: $13 \mathrm{~nm}, 795-808 \mathrm{~nm}$ ), combination bands

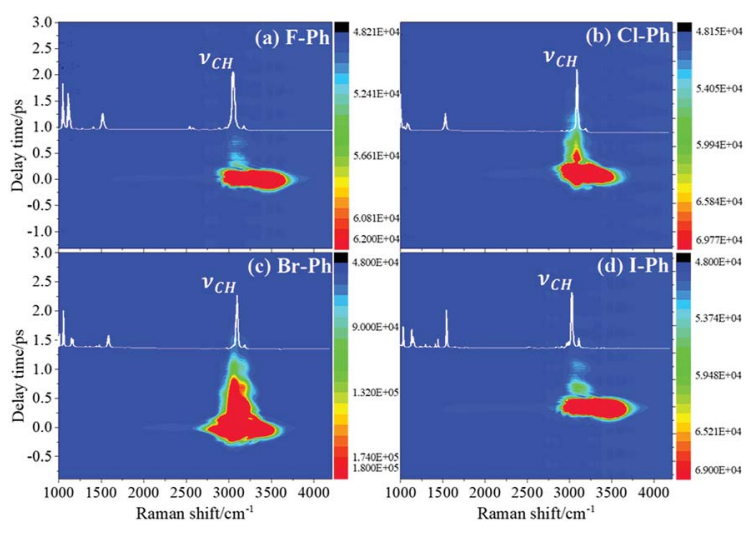

Fig. 1 The $\mathrm{C}-\mathrm{H}$ stretching vibrational modes of aryl halides are selectively excited, intramolecular energy flow to combination bands. Aryl halides: (a) F-Ph, (b) $\mathrm{Cl}-\mathrm{Ph}$, (c) $\mathrm{Br}-\mathrm{Ph}$, and (d) I-Ph. arising from vibrational energy redistribution are shown in Fig. 1(a)-(d), respectively. The high frequency $\mathrm{C}-\mathrm{H}$ stretching vibrational modes around $3070 \mathrm{~cm}^{-1}$ of aryl halides are excited instantaneously. It is found that the intramolecular vibrational energy flow to the lower frequency substituent-dependent modes $\nu_{\mathrm{CX}^{1}} \sim 1080 \mathrm{~cm}^{-1}, \nu_{\mathrm{CC}}$ (in range from $1320 \mathrm{~cm}^{-1}$ to $1590 \mathrm{~cm}^{-1}$ ) and $\beta_{\mathrm{CH}}$ (in range from $1000 \mathrm{~cm}^{-1}$ to $1290 \mathrm{~cm}^{-1}$ ). Moreover, the signal at $2230 \mathrm{~cm}^{-1}$ is not fundamental modes, it emerges due to the Fermi resonance of $\nu_{\mathrm{CX}^{1}} \sim 1080 \mathrm{~cm}^{-1}$ and $\beta_{\mathrm{CH}} \sim 1150 \mathrm{~cm}^{-1}$ modes, another combination band at $2500 \mathrm{~cm}^{-1}$ emerges due to the Fermi resonance of $\nu_{\mathrm{CC}} \sim$ $1450 \mathrm{~cm}^{-1}$ and $\beta_{\mathrm{CH}} \sim 1066 \mathrm{~cm}^{-1}$ modes. ${ }^{5,10}$ Time-domain CARS spectra of fluorobenzene ( $\mathrm{F}-\mathrm{Ph})$, chlorobenzene (Cl-Ph), bromobenzene $(\mathrm{Br}-\mathrm{Ph})$, and iodobenzene (I-Ph) are shown in Fig. 1(a)-(d), respectively. Quantum beats in CARS spectra are derived from vibrational coupling of $\mathrm{C}-\mathrm{H}$ stretching modes and combination bands. Through Fourier analysis, the dynamic characteristics of the modes or combination bands involved in vibrational coupling can be extracted.

FT power spectra contain vibrational couplings information of high frequency $\mathrm{C}-\mathrm{H}$ stretching vibrational modes and lower frequency combination bands which are located outside of the direct excitation region, as shown in Fig. 2. Peak positions in the FT power spectra indicate the frequency difference between the two neighbouring modes. Fig. 2(a)-(d) correspond to the Fourier analysis of CARS signals of $\mathrm{F}-\mathrm{Ph}, \mathrm{Cl}-\mathrm{Ph}, \mathrm{Br}-\mathrm{Ph}$ and $\mathrm{I}-\mathrm{Ph}$, respectively. The peaks of Fourier transform (FT) power spectra that marked by $\mathrm{Q}_{i}$ are corresponding to the vibrational coupling of high frequency $\mathrm{C}-\mathrm{H}$ stretching vibrational modes and combination bands. Detailed information of coherent coupling among these relevant modes and their assignments are listed in ESI (Table $1 \dagger$ ), frequency difference between the identified vibrational modes matches well with the results from the FT spectra of aryl halides.

Combining the time-domain CARS spectra and its FT power spectra, vibrational couplings between high frequency $\mathrm{C}-\mathrm{H}$ stretching vibrational modes are confirmed. Meanwhile lower frequency combination bands $\nu_{\mathrm{CM}^{1}} \sim 2230 \mathrm{~cm}^{-1}$ and $\nu_{\mathrm{CM}^{2}} \sim$ $2500 \mathrm{~cm}^{-1}$ which are secondly excited by vibrational energy redistribution are also identified.

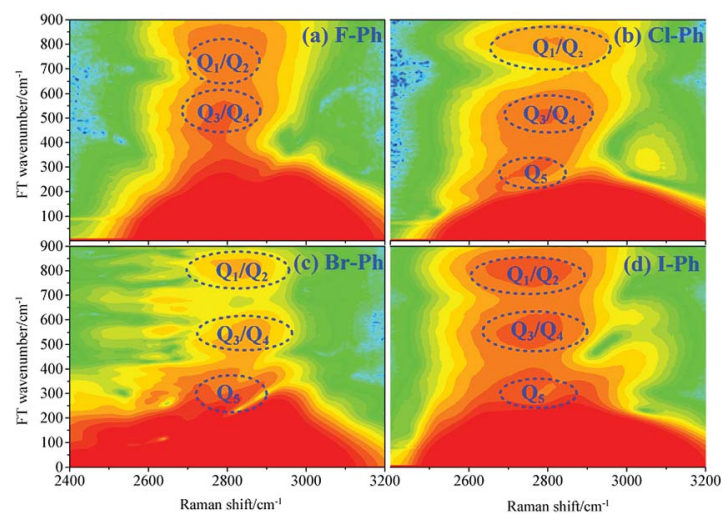

Fig. 2 FT power spectra of the $\mathrm{C}-\mathrm{H}$ stretching vibrational modes and combination bands. Aryl halides: (a) F-Ph, (b) $\mathrm{Cl}-\mathrm{Ph}$, (c) $\mathrm{Br}-\mathrm{Ph}$, and (d) I-Ph. 


\section{Selective excitation of the $\mathrm{C}-\mathrm{C}$ stretching vibrational modes}

Vibrational modes in the frequency range from 1362 to $1788 \mathrm{~cm}^{-1}$ are selectively excited by a pair of pump $(711 \mathrm{~nm}$, FHWM: $11 \mathrm{~nm}, 706-717 \mathrm{~nm}$ ) and Stokes pulses (800 nm, FHWM: $13 \mathrm{~nm}, 795-808 \mathrm{~nm}$ ), combination bands arising from intramolecular energy redistribution are shown in Fig. 3. There are five $\mathrm{C}-\mathrm{C}$ stretching vibrational modes and all of them are excited simultaneously. Fig. 3(a)-(d) are the time-resolved CARS spectra of $\mathrm{F}-\mathrm{Ph}, \mathrm{Cl}-\mathrm{Ph}, \mathrm{Br}-\mathrm{Ph}$ and $\mathrm{I}-\mathrm{Ph}$, respectively. The whitecoded lines represent the experimental spontaneous Raman spectra of aryl halides. Combination bands $\left(\nu_{\mathrm{CM}^{1}} \sim 2230 \mathrm{~cm}^{-1}\right.$ and $\left.\nu_{\mathrm{CM}^{2}} \sim 2500 \mathrm{~cm}^{-1}\right)$ and in-plane C-H deformations $\left(\beta_{\mathrm{CH}} \sim\right.$ $1150 \mathrm{~cm}^{-1}$ ) modes emerge in the CARS spectra even though they are outside of direct excitation range. It denotes vibrational energy can flow from lower frequency modes to higher ones. Vibrational couplings will lead to quantum beats in the timeresolved CARS spectra, and the characteristic frequencies of beats can be extracted through Fourier analysis.

Fourier analysis is performed to identify the vibrational modes involved in coherent coupling in the direct excitation region around $1500 \mathrm{~cm}^{-1}$, as shown in Fig. 4. The peaks of FT power spectra that marked by $\mathrm{Q}_{i}(i=1-6)$ are corresponding to the vibrational coupling of $\mathrm{C}-\mathrm{C}$ stretching vibrational modes and in-plane $\mathrm{C}-\mathrm{H}$ deformations $\left(\beta_{\mathrm{CH}} \sim 1150 \mathrm{~cm}^{-1}\right)$ modes of aryl halides, as described in Fig. 4(a)-(d). Detailed assignments of coherent couplings of $\mathrm{C}-\mathrm{C}$ stretching vibrational modes and in-plane $\mathrm{C}-\mathrm{H}$ deformations modes are listed in ESI (Table $2 \dagger$ ). The vibrational couplings of $\mathrm{C}-\mathrm{C}$ stretching $\nu_{\mathrm{CC}}$ (in range from $1320 \mathrm{~cm}^{-1}$ to $1590 \mathrm{~cm}^{-1}$ ) and $\beta_{\mathrm{CH}}$ (in range from $1000 \mathrm{~cm}^{-1}$ to $1290 \mathrm{~cm}^{-1}$ ) can be identified easily.

Combining the results of time-domain CARS spectra of aryl halides and their FT power spectra, it can be confirmed that the vibrational energy can flow from $\mathrm{C}-\mathrm{C}$ stretching vibrational modes to combination bands and the in-plane $\mathrm{C}-\mathrm{H}$ deformation modes. Vibrational modes of C-C stretching $\nu_{\mathrm{CC}}$ (in range from $1320 \mathrm{~cm}^{-1}$ to $1590 \mathrm{~cm}^{-1}, A_{1}$ or $B_{1}$ symmetry) involved in couplings are confirmed.
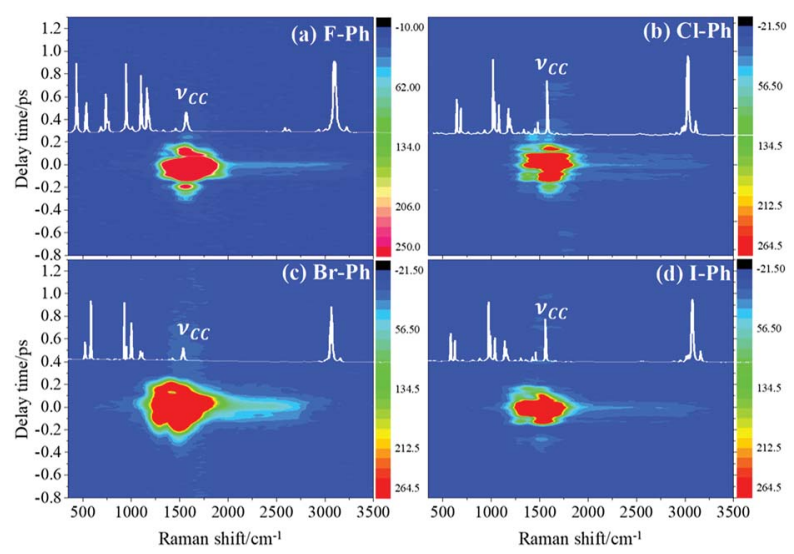

Fig. 3 The $\mathrm{C}-\mathrm{C}$ stretching vibrational modes are selectively excited, intramolecular energy flow to combination bands and in-plane $\mathrm{C}-\mathrm{H}$ deformations modes $\left(\beta_{\mathrm{CH}} \sim 1150 \mathrm{~cm}^{-1}\right)$. Aryl halides: (a) $\mathrm{F}-\mathrm{Ph}$, (b) $\mathrm{Cl}-$ $\mathrm{Ph}$, (c) $\mathrm{Br}-\mathrm{Ph}$, and (d) I-Ph.

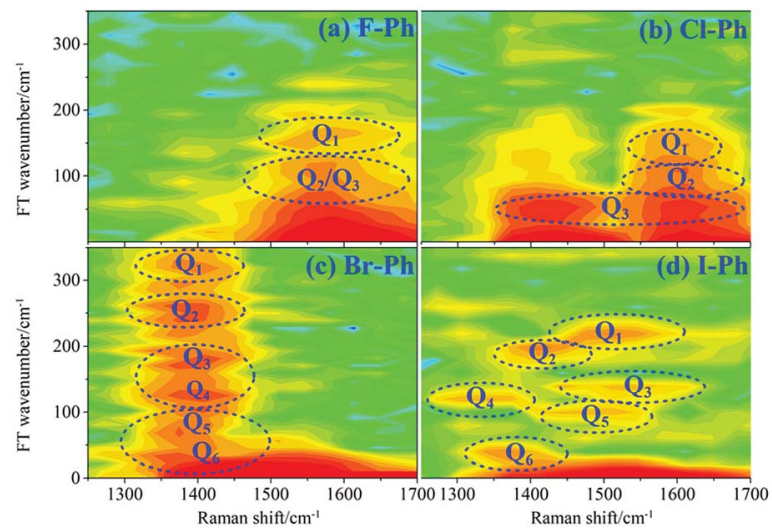

Fig. 4 FT power spectra of $\mathrm{C}-\mathrm{C}$ stretching vibrational modes $\nu_{\mathrm{CC}}$ $\left(\sim 1580 \mathrm{~cm}^{-1}\right)$ and in-plane $\mathrm{C}-\mathrm{H}$ deformations modes $\left(\beta_{\mathrm{CH}} \sim\right.$ $1150 \mathrm{~cm}^{-1}$ ). Aryl halides (a) F-Ph, (b) $\mathrm{Cl}-\mathrm{Ph}$, (c) $\mathrm{Br}-\mathrm{Ph}$, and (d) I-Ph.

\section{Selective excitation of the substituent-dependent mode $\nu_{\mathrm{CX}^{1}}$}

Vibrational modes in the frequency range from $880 \mathrm{~cm}^{-1}$ to $1303 \mathrm{~cm}^{-1}$ are directly excited by pump (745 nm, FHWM: $12 \mathrm{~nm}, 739-751 \mathrm{~nm}$ ) and Stokes pulses (800 nm, FHWM: $13.4 \mathrm{~nm}, 795-808 \mathrm{~nm})$, the excited modes including the substituent-dependent mode $\nu_{\mathrm{CX}^{1}}\left(\sim 1080 \mathrm{~cm}^{-1}\right), \beta_{\mathrm{CH}}$ (in range from $1000 \mathrm{~cm}^{-1}$ to $1290 \mathrm{~cm}^{-1}$ ) and $\gamma_{\mathrm{CH}}$ (in range from $880 \mathrm{~cm}^{-1}$ to $980 \mathrm{~cm}^{-1}$ ). Intramolecular energy flow from parent modes to daughter modes of $\mathrm{C}-\mathrm{C}$ stretching in liquid aryl halides are observed clearly in Fig. 5. Parent modes: vibrational modes which are directly excited by the pump and Stokes pulses; daughter modes: vibrational modes accepting the vibrational energy through intramolecular vibrational energy redistribution. The white-coded lines represent the experimental Raman spectra of aryl halides. The contour plots of Fig. 5(a)-(d) are the time-resolved CARS spectra of $\mathrm{F}-\mathrm{Ph}, \mathrm{Cl}-\mathrm{Ph}, \mathrm{Br}-\mathrm{Ph}$ and $\mathrm{I}-\mathrm{Ph}$, respectively. Signals outside of excitation range emerge around $1580 \mathrm{~cm}^{-1}$ are indirectly excited through intramolecular energy redistribution, and they correspond to the $\mathrm{C}-\mathrm{C}$ stretching vibrational modes. Even though the lower frequency modes cannot be distinguished directly due to the low resolution in
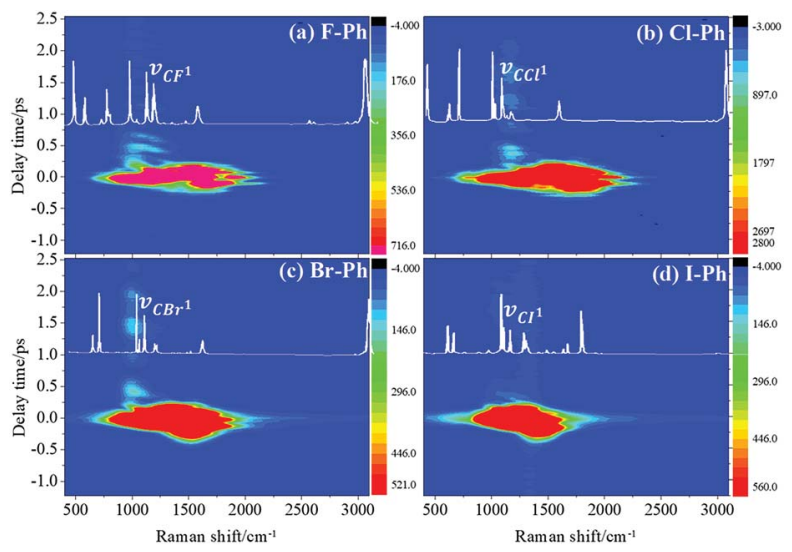

Fig. 5 Substituent-dependent mode $\nu_{\mathrm{CX}}\left(\sim 1080 \mathrm{~cm}^{-1}\right)$ are selectively excited, the vibrational energy flow to the $\mathrm{C}-\mathrm{C}$ stretching vibrational modes $\nu_{\mathrm{CC}}$ (in range from $1320 \mathrm{~cm}^{-1}$ to $1590 \mathrm{~cm}^{-1}$ ). Aryl halides: (a) F$\mathrm{Ph}$, (b) $\mathrm{Cl}-\mathrm{Ph}$, (c) $\mathrm{Br}-\mathrm{Ph}$, and (d) I-Ph. 


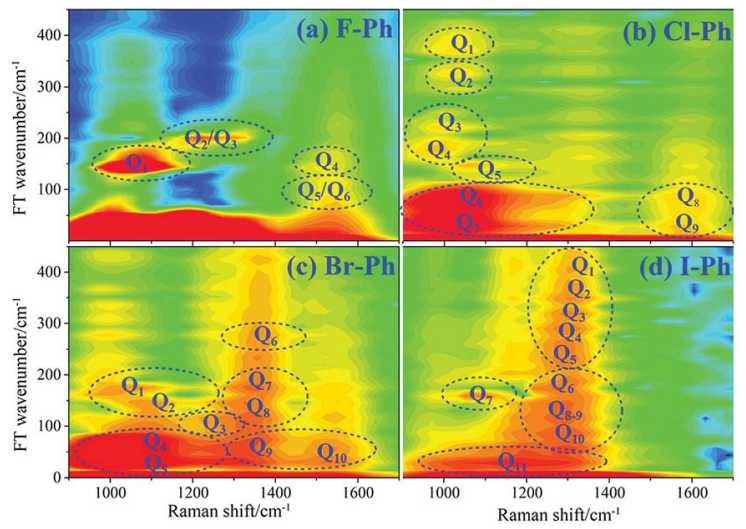

Fig. $6 \mathrm{FT}$ power spectra of the modes in range from 900 to $1600 \mathrm{~cm}^{-1}$. Aryl halides (a) F-Ph, (b) $\mathrm{Cl}-\mathrm{Ph}$, (c) $\mathrm{Br}-\mathrm{Ph}$, and (d) I-Ph.

frequency domain in ultrafast CARS spectra, they can be extracted through Fourier transform of the time-domain CARS spectra.

FT power spectra contain vibrational couplings information of vibrational modes that range from 900 to $1300 \mathrm{~cm}^{-1}$ and C-C stretching modes which are located outside of the directly excitation region, as shown in Fig. 6 . The peaks position in the FT power spectra indicate the frequency difference between two neighbouring modes and mark by $\mathrm{Q}_{i}(i=1-11)$, Fig. 6(a) represent the FT power spectra of $\mathrm{F}-\mathrm{Ph}, \mathrm{Cl}-\mathrm{Ph}, \mathrm{Br}-\mathrm{Ph}$ and $\mathrm{I}-\mathrm{Ph}$, respectively. Detailed information of coherent couplings of $\nu_{\mathrm{CX}^{1}}$ ( $\left.\sim 1080 \mathrm{~cm}^{-1}\right), \beta_{\mathrm{CH}}$ (in range from $1000 \mathrm{~cm}^{-1}$ to $\left.1290 \mathrm{~cm}^{-1}\right), \gamma_{\mathrm{CH}}$ (in range from $730 \mathrm{~cm}^{-1}$ to $980 \mathrm{~cm}^{-1}$ ) and $\nu_{\mathrm{CC}}$ (in range from $1320 \mathrm{~cm}^{-1}$ to $1590 \mathrm{~cm}^{-1}$ ) are listed in ESI (Table $3 \dagger$ ).

According to the results of the time-domain CARS spectra and its FT power spectra, the vibrational couplings of $\nu_{\mathrm{CX}^{1}}$ $\left(\sim 1080 \mathrm{~cm}^{-1}\right), \beta_{\mathrm{CH}}$ (in range from $1000 \mathrm{~cm}^{-1}$ to $1290 \mathrm{~cm}^{-1}$ ) and $\gamma_{\mathrm{CH}}$ (in range from $730 \mathrm{~cm}^{-1}$ to $980 \mathrm{~cm}^{-1}$ ) are confirmed, meanwhile C-C stretching modes $\nu_{\mathrm{CC}}$ (in range from $1320 \mathrm{~cm}^{-1}$ to $1590 \mathrm{~cm}^{-1}$ ) which are excited secondly by vibrational energy redistribution are also identified.

\section{Selective excitation of the substituent-dependent mode $\nu_{\mathrm{CX}^{2}}$}

Vibrational modes in the frequency range from 418 to $833 \mathrm{~cm}^{-1}$ are selectively excited by a pair of pump (763 nm, FHWM: $12 \mathrm{~nm}$, 757-769 nm) and Stokes pulses (800 nm, FHWM: $13 \mathrm{~nm}, 795-808$ $\mathrm{nm})$. The lower frequency substituent-dependent mode $\nu_{\mathrm{CX}^{2}} \sim$ $700 \mathrm{~cm}^{-1}, \gamma_{\mathrm{CH}}$ (in range from $730 \mathrm{~cm}^{-1}$ to $833 \mathrm{~cm}^{-1}$ ) and $\phi_{\mathrm{CC}} \sim$ $600 \mathrm{~cm}^{-1}$ are excited coherently, as shown in Fig. 7. The CARS spectra of $\mathrm{F}-\mathrm{Ph}, \mathrm{Cl}-\mathrm{Ph}, \mathrm{Br}-\mathrm{Ph}$, and $\mathrm{I}-\mathrm{Ph}$ are presented in Fig. 7(a)-(d), respectively. The vibrational modes of $\beta_{\mathrm{CH}}$ (in range from $1000 \mathrm{~cm}^{-1}$ to $1290 \mathrm{~cm}^{-1}$ ) and $\nu_{\mathrm{CX}^{1}} \sim 1080 \mathrm{~cm}^{-1}$ appear in the CARS spectra even though they are outside of the direct excitation region. This means the vibrational energy can flow from lower frequency modes to higher frequency ones.

FT power spectra is performed to identify the vibrational modes involved in their coherent couplings in the direct excitation region around $700 \mathrm{~cm}^{-1}$, and in the second excitation region from $900 \mathrm{~cm}^{-1}$ to $1200 \mathrm{~cm}^{-1}$, as shown in Fig. 8. The peaks of FT power spectra are marked by $\mathrm{Q}_{i}(i=1-10)$ and

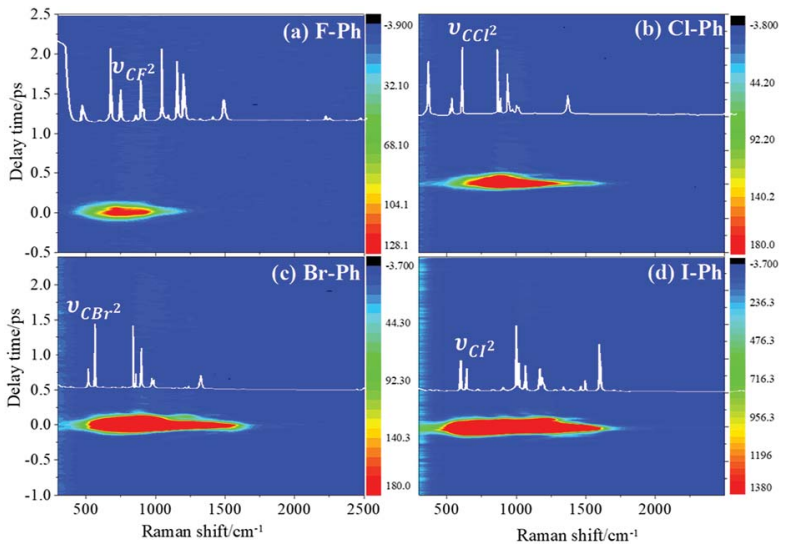

Fig. 7 Vibrational modes of $\nu_{\mathrm{CX}} \sim 700 \mathrm{~cm}^{-1}$ is selectively excited, intramolecular energy flow to neighbouring modes $\beta_{\mathrm{CH}} \sim 1150 \mathrm{~cm}^{-1}$ and $\nu_{\mathrm{CX}} \sim 1080 \mathrm{~cm}^{-1}$. Aryl halides: (a) $\mathrm{F}-\mathrm{Ph}$, (b) $\mathrm{Cl}-\mathrm{Ph}$, (c) $\mathrm{Br}-\mathrm{Ph}$, and (d) I-Ph.

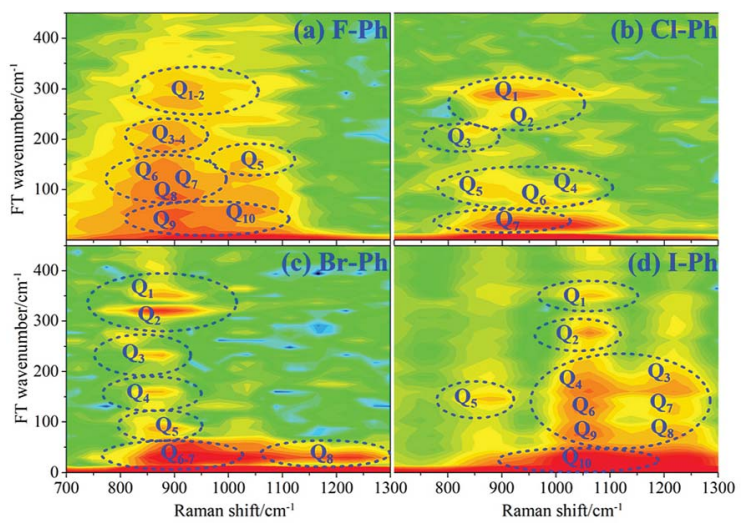

Fig. 8 FT power spectra of dominant excitation modes centred around $700 \mathrm{~cm}^{-1}$ and $1080 \mathrm{~cm}^{-1}$. Aryl halides: (a) $\mathrm{F}-\mathrm{Ph}$, (b) $\mathrm{Cl}-\mathrm{Ph}$, (c) $\mathrm{Br}-\mathrm{Ph}$, and (d) I-Ph.

correspond to the vibrational couplings of substitution modes $\nu_{\mathrm{CX}^{2}} \sim 700 \mathrm{~cm}^{-1}, \beta_{\mathrm{CH}}$ (in range from $1000 \mathrm{~cm}^{-1}$ to $1290 \mathrm{~cm}^{-1}$ ) and $\nu_{\mathrm{CX}^{1}} \sim 1080 \mathrm{~cm}^{-1}$ of aryl halides, as described in Fig. 8(a)(d). Detailed assignments of coherent couplings of $\nu_{\mathrm{CX}^{2}} \sim$ $700 \mathrm{~cm}^{-1}, \gamma_{\mathrm{CH}}$ (in range from $730 \mathrm{~cm}^{-1}$ to $980 \mathrm{~cm}^{-1}$ ) and $\phi_{\mathrm{CC}} \sim$ $600 \mathrm{~cm}^{-1}$ are listed in ESI (Table $4 \dagger$ ).

According to the analysis of the time-domain CARS spectra and its FT power spectra of aryl halides, it can be found that substitution modes $\nu_{\mathrm{CX}^{2}} \sim 700 \mathrm{~cm}^{-1}$, out-plane C-H deformation modes $\gamma_{\mathrm{CH}}$ (in range from $730 \mathrm{~cm}^{-1}$ to $980 \mathrm{~cm}^{-1}$ ) and out-plane ring deformation modes $\phi_{\mathrm{CC}} \sim 600 \mathrm{~cm}^{-1}$ are selectively excited, neighbouring modes $\beta_{\mathrm{CH}}$ (in range from $1000 \mathrm{~cm}^{-1}$ to $1290 \mathrm{~cm}^{-1}$ ) and $\nu_{\mathrm{CX}^{1}} \sim 1080 \mathrm{~cm}^{-1}$ outside of excitation range are emerged via vibrational energy redistribution.

\section{Intramolecular energy redistribution processes of the liquid aryl halides}

The above findings have implications for ultrafast physicochemical behaviours of the aryl halides and the characteristics 


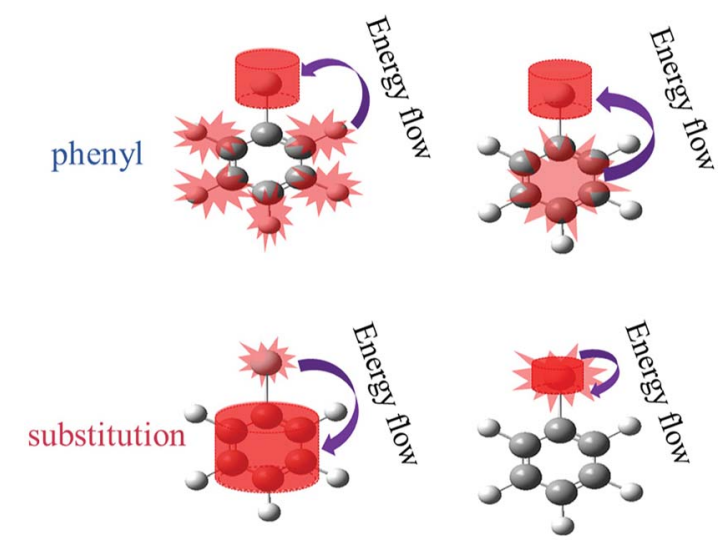

Fig. 9 The parent modes are selective excited, vibrational energy flow to the daughter mode. The upper two plots displayed the excitation modes of the phenyl $\left(\nu_{\mathrm{CH}}\right.$ and $\left.\nu_{\mathrm{CC}}\right)$; the lower ones displayed the excitation modes of the substituent-dependent modes $\left(\nu_{\mathrm{CX}} \text { and } \nu_{\mathrm{CX}}\right)^{2}$, the purple arrows represented the direction of intramolecular energy redistribution.

of intramolecular energy redistribution can be summarized as follows:

(1) The directionality. Intramolecular energy flow from the parent modes to daughter modes are described in Fig. 9. Vibrational energy can flow from high frequency $\mathrm{C}-\mathrm{H}$ stretching vibrational modes $\left(\nu_{\mathrm{CH}} \sim 3070 \mathrm{~cm}^{-1}\right)$ to the combination bands $\nu_{\mathrm{CM}^{1}} \sim 2230 \mathrm{~cm}^{-1}$ and $\nu_{\mathrm{CM}^{2}} \sim 2500 \mathrm{~cm}^{-1}$; from C-C stretching vibrational modes $\nu_{\mathrm{CC}} \sim 1580 \mathrm{~cm}^{-1}$ to the combination bands $\nu_{\mathrm{CM}^{1}} \sim 2230 \mathrm{~cm}^{-1}$ and $\nu_{\mathrm{CM}^{2}} \sim 2500 \mathrm{~cm}^{-1}$; from substituentdependent mode $\nu_{\mathrm{CX}^{1}} \sim 1080 \mathrm{~cm}^{-1}$ to the $\mathrm{C}-\mathrm{C}$ stretching vibrational modes $\nu_{\mathrm{CC}}$ (in range from $1320 \mathrm{~cm}^{-1}$ to $1590 \mathrm{~cm}^{-1}$ ); from substituent-dependent modes $\nu_{\mathrm{CX}^{2}} \sim 700 \mathrm{~cm}^{-1}$ to the substitution mode $\nu_{\mathrm{CX}^{1}} \sim 1080 \mathrm{~cm}^{-1}$. The up-hill energy flow from lower frequency modes to higher ones can be confirmed.

(2) Symmetry selection rule. Intramolecular energy redistribution occurs only between the parent modes and the daughter ones that have the same vibrational symmetry. For example, while $\mathrm{C}-\mathrm{H}$ stretching vibrational modes $\nu_{\mathrm{CH}} \sim$ $3070 \mathrm{~cm}^{-1}$ are selectively excited, vibrational energy flows to combination bands $\nu_{\mathrm{CM}^{1}}$ and $\nu_{\mathrm{CM}^{2}}\left(A_{1}\right.$ and $B_{1}$ symmetry); while C-C stretching vibrational modes $\nu_{\mathrm{CC}} \sim 1580 \mathrm{~cm}^{-1}\left(A_{1}\right.$ or $B_{1}$ symmetry) are selectively excited, vibrational energy flows to the combination bands $\nu_{\mathrm{CM}^{1}}$ and $\nu_{\mathrm{CM}^{2}}$; while substituent-dependent mode $\nu_{\mathrm{CX}^{1}} \sim 1100 \mathrm{~cm}^{-1}$ ( $A_{1}$ symmetry) is selectively excited, energy flows to vibrational modes of $\mathrm{C}-\mathrm{C}$ stretching vibrational modes ( $A_{1}$ or $B_{1}$ symmetry); while substituent-dependent mode $\nu_{\mathrm{CX}^{2}} \sim 700 \mathrm{~cm}^{-1}$ ( $A_{1}$ symmetry) is selectively excited, energy flows to $\nu_{\mathrm{CX}^{1}}$ ( $A_{1}$ symmetry). A rule can be found that intramolecular energy redistribution processes is related to vibrational symmetry. Specifically, the energy flow only occurs among these relevant modes or bands with the same vibrational symmetry.

(3) Mass-dependence of intramolecular energy redistribution efficiency. It is amazing that the vibrational energy transfer between high frequency modes and lower ones is asymmetric, as shown in Fig. 10. Energy redistribution/transfer efficiency is

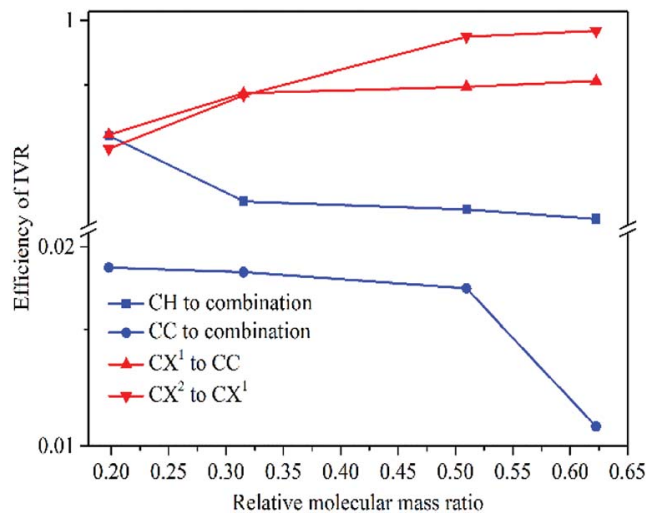

Fig. 10 Scatterplot of vibrational energy redistribution efficiencies.

defined by the intensity ratio between daughter modes and parent modes. The horizontal and vertical axes are corresponding to relative molecular mass ratio (the ratio of the halogen to the whole molecule) and the efficiency, respectively.

(1) While substituent-dependent modes $\left(\nu_{\mathrm{CX}^{1}}\right.$ and $\left.\nu_{\mathrm{CX}^{2}}\right)$ are selectively excited, vibrational energy flow from substituentdependent modes to phenyl modes $(\mathrm{CH}$ stretching and $\mathrm{CC}$ stretching), the efficiencies locate on the upper of the $2 \mathrm{D}$ scattering plot (red-coded symbol lines). While phenyl modes $\left(\nu_{\mathrm{CH}}\right.$ and $\left.\nu_{\mathrm{CC}}\right)$ of the aryl halides are selectively excited, vibrational energy flow from phenyl mode to substituent-dependent modes $\left(\nu_{\mathrm{CX}^{1}}\right.$ and $\left.\nu_{\mathrm{CX}^{2}}\right)$, the efficiencies locate on the lower of the 2D scattering plot (blue-coded symbol lines). It is found that the efficiencies of vibrational energy transfer from substituentdependent modes to phenyl modes are bigger than that of the inverse.

(2) Efficiency can be controlled by varying the mass ratio of the halide in the aryl halide molecules. On one hand, while the substituent-dependent modes $\left(\nu_{\mathrm{CX}^{1}}\right.$ and $\left.\nu_{\mathrm{CX}^{2}}\right)$ are selectively excited, efficiencies of vibrational energy redistribution are increased with increasing relative molecular mass ratio $\left(k_{\mathrm{I}-\mathrm{Ph}}>\right.$ $\left.k_{\mathrm{Br}-\mathrm{Ph}}>k_{\mathrm{Cl}-\mathrm{Ph}}>k_{\mathrm{F}-\mathrm{Ph}}\right)$. On the other hand, while the phenyl modes $\left(\nu_{\mathrm{CH}}\right.$ and $\left.\nu_{\mathrm{CC}}\right)$ of the aryl halides are selectively excited, efficiencies of vibrational energy redistribution are decreased with the increasing relative molecular mass ratio $\left(k_{\mathrm{I}-\mathrm{Ph}}<k_{\mathrm{Br}-\mathrm{Ph}}\right.$ $\left.<k_{\mathrm{Cl}-\mathrm{Ph}}<k_{\mathrm{F}-\mathrm{Ph}}\right)$.

The mass of phenyl is smaller than halides, the anharmonic couplings among these relevant modes are different and the efficiencies of vibrational energy redistribution are likely to be affected. While the heavier substitution-dependent modes are selectively excited, vibrational energy flow to lighter phenyl modes with larger amplitude oscillations, anharmonic coupling is more likely to happen and the efficiencies show a monotonic increase tendency with increasing mass of substituent of aryl halides. While the lighter phenyl modes are selectively excited, vibrational energy flow to substitutiondependent modes with the smaller amplitude oscillations, the anharmonic coupling is less likely to happen and the efficiencies show a monotonic decrease tendency with increasing mass of substituents. 


\section{Conclusions}

Time-resolved CARS technique is performed to selectively excite parent modes and detect the daughter modes of aryl halides. With the help of Fourier analysis, vibrational modes that involved in the ultrafast coherent coupling are determined. Moreover, it is found that intramolecular energy flow is counterintuitive, especially the efficiencies of up-hill vibrational energy redistribution from lower frequency modes to higher ones are always larger than that of the opposite direction. Vibrational excitation on phenyl modes $\left(\nu_{\mathrm{CH}}\right.$ and $\left.\nu_{\mathrm{CC}}\right)$, intramolecular energy redistribution efficiencies decrease with the increasing halide mass; on the contrary, vibrational excitation on the substituent-dependent modes $\left(\nu_{\mathrm{CX}^{1}}\right.$ and $\left.\nu_{\mathrm{CX}^{2}}\right)$, efficiencies increase with the increasing halide mass. It is noting that halide mass can control intramolecular energy redistribution efficiencies, and these results provide implications of substituent mass effects on photochemical reactions.

\section{Conflicts of interest}

There are no conflicts to declare.

\section{Acknowledgements}

This work is supported by the Science Challenging Program (grant number TZ2016001) and the National Natural Science Foundation of China (grant number 21673211).

\section{Notes and references}

1 T. M. Krygowski and B. T. Stepień, ChemInform, 2005, 105, 3482 .
2 L. Guan, M. G. Holl, C. R. Pitts, M. D. Struble, M. A. Siegler and T. Lectka, J. Am. Chem. Soc., 2017, 139, 14913.

3 G. Szabó and L. Nyulászi, Struct. Chem., 2017, 28, 1-11.

4 M. Huang, Z. Luo, T. Zhu, J. Chen, J. Z. Zhang and F. Xia, RSC Adv., 2017, 7, 51521-51527.

5 B. C. Pein, Y. Sun and D. D. Dlott, J. Phys. Chem. B, 2013, 117, 10898-10904.

6 B. C. Pein, Y. Sun and D. D. Dlott, J. Phys. Chem. A, 2013, 117, 6066-6072.

7 K. Okamoto, N. Nomura, R. Fujiyoshi, K. Umegaki, H. Yamamoto, K. Kobayashi and T. Kozawa, J. Phys. Chem. A, 2017, 121, 9458-9465.

8 R. V. Viesser, L. C. Ducati, C. F. Tormena and J. Autschbach, Phys. Chem. Chem. Phys., 2018, 20, 11247-11259.

9 I. V. Omelchenko, O. V. Shishkin, L. Gorb, F. C. Hill and J. Leszczynski, Struct. Chem., 2012, 23, 1585-1597.

10 A. M. Gardner and T. G. Wright, J. Chem. Phys., 2011, 135, 114305.

11 N. Levy, J. S. Shpilman, H. C. Honig, D. T. Major and L. Elbaz, Chem. Commun., 2017, 53, 12942.

12 W. Shen, W. Zhang and C. Zhu, Phys. Chem. Chem. Phys., 2017, 19, 23532.

13 X. Liu, W. Zhang, Y. Song, G. Yu, Z. Zheng, Y. Zeng, Z. Lv, H. Song and Y. Yang, J. Phys. Chem. A, 2017, 121, 4948-4952.

14 D. H. Whiffen, J. Chem. Soc., 1956, 4, 8.

15 B. C. Pein, N. H. Seong and D. D. Dlott, J. Phys. Chem. A, 2010, 114, 10500-10507.

16 S. Singh, D. K. Singh, S. K. Srivastava and B. P. Asthana, Vib. Spectrosc., 2011, 56, 26-33.

17 J. R. Scherer and J. C. Evans, Spectrochim. Acta, 1963, 19, 1739-1775. 